OPEN ACCESS

Edited by:

Shumin Duan,

Zhejiang University, China

Reviewed by:

Amit Agarwal,

Johns Hopkins University, USA

David Peter Gavin,

Jesse Brown VA Medical Center,

USA

Frank Kirchhoff,

Saarland University, Germany

*Correspondence: Junlin Yang

yjl8121@yahoo.com

Mengsheng Qiu

m0qiu001@yahoo.com

Received: 20 December 2016 Accepted: 28 March 2017 Published: 11 April 2017

Citation:

Yang J, Cheng X, Qi J, Xie B, Zhao X, Zheng $K$, Zhang Z and Qiu M (2017) EGF Enhances Oligodendrogenesis from Glial Progenitor Cells.

Front. Mol. Neurosci. 10:106. doi: 10.3389/fnmol.2017.00106

\section{EGF Enhances Oligodendrogenesis from Glial Progenitor Cells}

\author{
Junlin Yang ${ }^{1 *}$, Xuejun Cheng ${ }^{1}$, Jiajun Qi $^{1}$, Binghua Xie ${ }^{1}$, Xiaofeng Zhao ${ }^{1}$, Kang Zheng ${ }^{1}$, \\ Zunyi Zhang ${ }^{1}$ and Mengsheng Qiu ${ }^{1,2 *}$
}

${ }^{1}$ The Institute of Developmental and Regenerative Biology, Zhejiang Key Laboratory of Organ Development and Regeneration, College of Life and Environment Sciences, Hangzhou Normal University, Hangzhou, China, ${ }^{2}$ Department of Anatomical Sciences and Neurobiology, University of Louisville, Louisville, KY, USA

Emerging evidence indicates that epidermal growth factor (EGF) signaling plays a positive role in myelin development and repair, but little is known about its biological effects on the early generation and differentiation of oligodendrocyte $(\mathrm{OL})$ lineage cells. In this study, we investigated the role of EGF in early OL development with isolated glial restricted precursor (GRP) cells. It was found that EGF collaborated with Platelet Derived Growth Factor-AA (PDGFaa) to promote the survival and self-renewal of GRP cells, but predisposed GRP cells to develop into $\mathrm{O}^{-}{ }^{-}$early-stage oligodendrocyte precursor cells (OPCs) in the absence of or PDGFaa. In OPCs, EGF synergized with PDGFaa to maintain their $\mathrm{O} 4$ negative antigenic phenotype. Upon PDGFaa withdrawal, EGF promoted the terminal differentiation of OPCs by reducing apoptosis and increasing the number of mature OLs. Together, these data revealed that EGF is an important mitogen to enhance oligodendroglial development.

Keywords: GRP cell, OPC, oligodendrocyte lineage, self-renewal, synergistic effect

\section{INTRODUCTION}

Oligodendrocytes (OLs) elaborate insulating myelin sheaths that wrap around axons to ensure the rapid conduction of nerve impulses and axonal survival (Qiu, 2013; Zhang et al., 2013; Blank and Prinz, 2014; Alizadeh et al., 2015; Rao and Pearse, 2016). Tripotential glial-restricted precursor (GRP) cells were initially found in embryonic spinal tissues (Herrera et al., 2001; Gregori et al., 2002; Wu et al., 2002; Cao et al., 2005; Phillips et al., 2012), as they can generate OLs and type I and type II astrocytes in vitro (Rao et al., 1998; Gregori et al., 2002; Yang et al., 2016) and in vivo (Herrera et al., 2001; Hill et al., 2004). The germination of GRP cells from neuroepithelial stem cells was viewed as the beginning of OL generation (Rao et al., 1998; Gregori et al., 2002). Tripotential GRP cells then generate bipotential oligodendrocyte precursor cells (OPCs) which are capable of differentiating into either OLs or type II astrocytes (Morath and Mayer-Pröschel, 2001; Gregori et al., 2002). OPCs proliferate and migrate throughout the CNS during late mouse embryonic development, and later differentiate into mature myelinating OLs (Fernandez et al., 2004; Cai et al., 2010; Chen et al., 2015).

Emerging evidence suggests that epidermal growth factor (EGF) signaling plays an important role in oligodendroglial development (Aguirre et al., 2007; Chong et al., 2008; Hu et al., 2010).

Abbreviations: bFGF, Basic fibroblast growth factor; EGF, Epidermal growth factor; EGFR, Epidermal growth factor receptor; GRP, Glial restricted precursor; OL, Oligodendrocyte; OPC, Oligodendrocyte precursor cells; PDGFaa, Platelet derived growth factor-AA. 
Loss-of-function of epidermal growth factor receptor (EGFR) reduced oligodendrogenesis in vivo (Aguirre et al., 2007); conversely, intraventricular infusion of EGF induced subventricular zone (SVZ) type B cells to migrate and differentiate into OLs (Gonzalez-Perez et al., 2009). More recently, it was shown that intranasal EGF treatment immediately after brain injury promoted oligodendrogenesis and remyelination (Scafidi et al., 2014). Although the importance of EGF signaling in the development of OL lineage has been established, it remains elusive at what stage of oligodendrogenesis EGF starts to function and how it regulates the development of OL lineage progression.

In this study, we used mouse GRP cells as the starting point to systematically investigate the role of EGF signaling in OL lineage development. It was found that cells of OL lineage were responsive to EGF at all developmental stages. In GRP cells, EGF promoted their proliferation and survival by augmenting their responsiveness to Platelet Derived Growth Factor-AA (PDGFaa) for self-renewal. In the absence of PDGFaa, EGF predisposed GRP cells to differentiate into $\mathrm{O}^{-}$early-stage OPCs. At OPC stage, EGF collaborated with PDGFaa to enhance OPC selfrenewal. Upon PDGFaa withdrawal, OPCs underwent terminal differentiation and EGF functioned to reduce apoptosis and increase the number of mature OLs.

\section{MATERIALS AND METHODS}

\section{Isolation and Culture of GRP Cells}

GRP cells were isolated from E13.5 C57BL/6 mouse spinal cord by A2B5 immunopanning as described elsewhere (Gregori et al., 2002), all experimental procedures were carried out in accordance with institutional guidelines for the care and use of laboratory animals, and the protocol was approved by the Animal Ethics Committee of Hangzhou Normal University, China. $\mathrm{A} 2 \mathrm{~B}^{+}$cells were then grown in glial basal medium $(\mathrm{DMEM} / \mathrm{F} 12,1 \times \mathrm{N} 2,1 \times \mathrm{B} 27,1 \times \mathrm{P} / \mathrm{S}$, and $0.1 \% \mathrm{w} / \mathrm{v} \mathrm{BSA}$, all from Gibco) supplemented with $10 \mathrm{ng} / \mathrm{ml}$ PDGFaa and EGF (Peprotech) on fibronectin/laminin coated 12-well plates at 2000 cells/well for mass culture experiments or on coated 24-well plated at 1000 cells/well for immunofluorescence staining. The immunostaining was performed 3 days after seeding using the standard protocols.

\section{Clonal Analysis and Sub-Cloning of GRP Cells}

Immunopurified $\mathrm{A} 2 \mathrm{~B}^{+}{ }^{+}$cells from E13.5 spinal tissues were adjusted to a cell density of 10 cells/ml with glial basal medium supplemented with $10 \mathrm{ng} / \mathrm{ml}$ EGF and PDGFaa, then the cell suspension was added into fibronectin/laminin-coated 96-well plates at $100 \mu \mathrm{l} /$ well, and wells with a single cell were marked for further culture. When primary clones were generated, 10 clones were randomly selected and only one was found to be $\mathrm{A} 2 \mathrm{~B} 5$ negative. Of the nine $\mathrm{A} 2 \mathrm{~B} 5^{+}$clones, three were subjected to differentiation potential analysis as indicated in the "Results" Section. The other six $\mathrm{A} 2 \mathrm{~B} 5^{+}$clones were used for sub-cloning analysis. Each clone was replated on three separate grid dishes at equal clonal density, and cultured in presence of EGF, PDGFaa or both. After 6 days, the numbers of secondary clones were scored.

\section{Cell Proliferation and Apoptosis Analysis}

For cell proliferation and apoptosis analysis, $1 \times 10^{4}$ cells were plated to each fibronectin/laminin-coated 24-well plates. Cell proliferation was analyzed by adding BrdU (Sigma) to a final concentration of $30 \mathrm{ng} / \mathrm{ml}$. Following $24 \mathrm{~h}$ of incorporation, cells were fixed in $4 \%$ paraformaldehyde at RT for $10 \mathrm{~min}$, and BrdU positive cells were detected by anti-BrdU immunostaining. For apoptosis assays, cells were fixed in $4 \%$ paraformaldehyde 3 days after replating and apoptotic cells were detected by TUNEL FITC Apoptosis Detection Kit (Vazyme Biotech). Positive cells were counted from three different areas of each well under fluorescence microscopy. The results were expressed as mean values and standard deviation.

\section{GRP Cell Development}

The function of EGF on the development of GRP cells into OPCs was confirmed by single-cell tracking clonal differentiation analysis as described previously (Gregori et al., 2002). Freshly immunopurified GRP cells from E13.5 spinal tissues were adjusted to a cell density of 10 cells $/ \mathrm{ml}$ with glial basal medium supplemented, then the cell suspension was added into fibronectin/laminin-coated 48 -well plates at $100 \mu \mathrm{l} /$ well in the presence of EGF and PDGFaa $(10 \mathrm{ng} / \mathrm{ml})$ for $24 \mathrm{~h}$ before being exposed to the factors as indicated in the "Results" Section wells with a single cell were marked for further culture. Since there is no specific immunological marker to distinguish GRP cells from OPCs, one candidate marker for distinguishing them is the $\mathrm{O} 4$ monoclonal antibody, which labels late-stage OPCs and OLs (Gard and Pfeiffer, 1990; Bansal et al., 1992). Therefore, the proportion of $\mathrm{O}^{+}$cells was used as a standard to estimate the differentiation of GRP cells.

\section{OPC Culture}

O4- early-stage OPCs were induced from GRP cells and plated on the fibronectin/laminin-coated plates and fed every other day with glial basal medium supplemented with EGF and PDGFaa. Because of the presence of contact inhibition, OPCs were plated more sparsely than GRP cells, and passaged more frequently. OPCs were plated at 1500 cells $/ \mathrm{cm}^{2}$ for mass culture, and 750 cells $/ \mathrm{cm}^{2}$ for differentiation experiments due to process growth.

\section{Western Immunoblotting}

Western blotting was carried out as previously described (Yang et al., 2009). Briefly, cells were lysed in sample buffer plus a cocktail of protease inhibitors (Roche). For each sample, $20 \mu \mathrm{g}$ of protein was used for electrophoresis in SDS-PAGE gel. Primary antibodies were used as follows; anti-rabbit PDGFR $\alpha$ (1:1000, Santa Cruz), anti- rabbit EGFR (1:200, Abcam), antirabbit Olig2 (1:1000, Millipore), anti-rabbit Nestin (1:5000, Covance) and anti-mouse MBP (1:1000, Abcam). Horseradish peroxidase (HRP)-conjugated secondary antibody (Promaga) was used at 1:2500. Chemiluminescent signals were detected by 
autoradiography using the ECL System (Amersham, Piscataway, NJ, USA).

\section{Immunocytochemical Analysis}

Immunocytochemical analysis was carried out as previously described (Cheng et al., 2017). Antibodies used include anti-mouse A2B5 IgM, anti-BrdU IgM, O4 IgM (1:1 dilution in DPBS $+10 \%$ goat serum), anti-mouse Olig2 (1:1000, Millipore), anti-mouse MBP (1:500, Abcam), anti- rabbit EGFR (1:200, Abcam), anti-mouse GFAP (1:300, Chemicon), anti-rabbit Nestin (1:2000, Covance), and anti-rabbit neurofilament (1:100, Sigma). The Alexa-488 or Alexa-594 conjugated secondary antibodies were obtained from Molecular Probes (Thermo fisher). The nucleic acid dye 4',6-diamidino-2-phenylindole (DAPI) was obtained from Roche.

\section{Statistical Analysis}

All quantitative data are presented as means \pm SD. Statistical significance of the difference was evaluated by Student's $t$-test. $P$-value $<0.05$ was considered statistically significant.

\section{RESULTS}

\section{EGF Enhances the Survival and Extensive Self-Renewal of GRP Cells in Culture}

To investigate the role of EGF on OL lineage development, we first immunopurified $\mathrm{A} 2 \mathrm{~B}^{+}$cells from E13.5 spinal cord tissues (Liu et al., 2002). These $\mathrm{A}^{2} \mathrm{~B}^{+}{ }^{+}$cells expressed typical GRP markers including PDGFR $\alpha$ (Rao et al., 1998), Olig2 (Zhao et al., 2009) and Nestin (Yoo and Wrathall, 2007; Figures 1A-D,I-Q), but not neuronal marker neurofilament (Tang et al., 2007; Rao and Pearse, 2016) and astrocyte marker GFAP (Sun et al., 2008; Sántha et al., 2016). The GRP cells were also immunoreactive for EGFR (Figures 1E-H,Q) suggesting their potential EGF-responsiveness. The effects of EGF on $\mathrm{A} 2 \mathrm{~B} 5^{+}$cell proliferation were investigated in culture exposed to different concentrations of EGF $(0,2.5,5,10,20$ and $40 \mathrm{ng} / \mathrm{ml}$ ) for $24 \mathrm{~h}$. BrdU labeling revealed a dose-dependent effect on cell division at the concentration range of $0-10 \mathrm{ng} / \mathrm{ml}$ (Figure 2A). However, no statistical differences were found in the percentage of $\mathrm{BrdU}^{+}$cells among the 10, 20 and $40 \mathrm{ng} / \mathrm{ml} \mathrm{EGF}$ groups (Figure 2A), indicating that cell proliferation plateaued at $10 \mathrm{ng} / \mathrm{ml}$. When we grew $\mathrm{A}_{2} \mathrm{~B}^{+}$cells for 7 days, EGF was found to promote the cell proliferation and survival as effectively as basic fibroblast growth factor (bFGF), but the percentages of $\mathrm{BrdU}^{+}$cells on $\mathrm{d} 3$ and $\mathrm{d} 7$ in EGF groups were higher than those of bFGF groups (Figures 2B,C). When GRP cells were treated with EGF and Erlotinib $\mathrm{HCl}$ (an antagonist of EGFR) simultaneously, the biological effects of EGF on promoting the division and survival was neutralized (Figures 2B,C), and the antigenic phenotypes were similar to those of the control (Figure 4B). T3 did not significantly enhance the proliferation of $\mathrm{A}_{2} \mathrm{~B}^{+}$cells compared to control group (Figure 2B), but it significantly reduced cell apoptosis (Figure $2 \mathrm{C}$ ), probably by promoting $\mathrm{A}_{2} \mathrm{~B}^{+}$cell differentiation into OLs (Figures 3D,E).
EGF also cooperated with PDGFaa to promote a vibrant proliferation of $\mathrm{A} 2 \mathrm{~B} 5^{+}$cells as bFGF + PDGFaa did (Figures 2B,C). As a result, EGF + PDGFaa stimulated A2B5 ${ }^{+}$ cells to divide continuously and form clones (Figures 2B, 3A). Three randomly chosen primary clones (EGF + PDGFaa treatment) were digested into single cells with trypsin and replated for antigen phenotyping and differentiation potential analysis. All clones from the freshly immunopurified $\mathrm{A} 2 \mathrm{~B}^{+}$ cells expressed the same antigens as described above. Cells grown in the presence of cholinergic neurotrophic factor (CNTF) and bFGF mainly yielded $\mathrm{A} \mathrm{B}^{+} / \mathrm{GFAP}^{+}$type II astrocytes, but $\mathrm{A}^{2} \mathrm{~B}^{-} / \mathrm{GFAP}^{+}$type I astrocytes in the presence of FBS (Figures 3B,C). When exposed to thyroid hormone T3 (Rodríguez-Peña, 1999) for 5 days, all clones gave rise to $\mathrm{MBP}^{+}$mature OLs with multiple interconnecting processes (Figures 3D,E; Shaw et al., 1981). No neurofilament ${ }^{+}$neurons were generated in these cultures. Thus, these $\mathrm{A} 2 \mathrm{~B}^{+}{ }^{+}$cells are the bona fide tripotential glial progenitor cells (GRP cells), with the potential to generate OLs and two distinct types of astrocytes (Gregori et al., 2002; Dadsetan et al., 2009; Haas et al., 2012).

Recloning experiments showed EGF and PDGFaa have a synergistic effect on the extensive self-renewal of GRP cells. After primary clones were amplified in EGF + PDGFaa for 10 days, randomly selected clones were digested into single cells and evenly plated at clonal density on three separate grid dishes, and then cultured in the presence of EGF, PDGFaa, or both. EGF and PDGFaa treatment yielded an average of 21 and 6 secondary clones, respectively. When these two factors were added together, the number of secondary clones reached an average of 51, far greater than the sum of individual factors (Figure 2D), suggesting that EGF synergized with PDGFaa in stimulating the clonal expansion of GRP cells. Secondary clones exhibited an identical pattern of antigen expressions to primary clones and can differentiate into $\mathrm{MBP}^{+}$OLs, $\mathrm{A} 2 \mathrm{~B}^{+}$ or $\mathrm{A} 2 \mathrm{~B} 5^{-}$astrocytes under corresponding environmental cues. Based on these observations, it is concluded that EGF signaling participated in the survival and extensive self-renewal of GRP cells.

\section{EGF Promoted the Generation of Early-Stage OPCs from GRP Clones}

Tripotential GRP cells are capable of developing into bipotential OPCs under appropriate signals (Gregori et al., 2002). To investigate how EGF signaling influences this transition process, we grew freshly immunopurified GRP cells at clonal density on 48-well plates for 5 days in the presence or absence of EGF, and then analyzed the formation of OPCs from a single GRP cell by tracking clonal differentiation (Figure $\mathbf{4 A}$ ) as described previously (Gregori et al., 2002). Since common GRP markers such as A2B5, Olig2, PDGFR $\alpha$ and Nestin were also positive for OPCs (Crang et al., 2004; Yang et al., 2016), only O4 monoclonal antibody can be used to define a secondary stage of OPC development (Sommer and Schachner, 1981; Chen et al., 2007; Dincman et al., 2012). In EGF group, $10.7 \% \pm 1.3 \%$ of the clones generated $\mathrm{O}^{+} / \mathrm{MBP}^{-}$cells (Figures $4 \mathrm{~A}, \mathrm{~B}$ ). While in the control group, GRP cells were unable to divide to form clones due to the lack of growth factors, so only a few single 

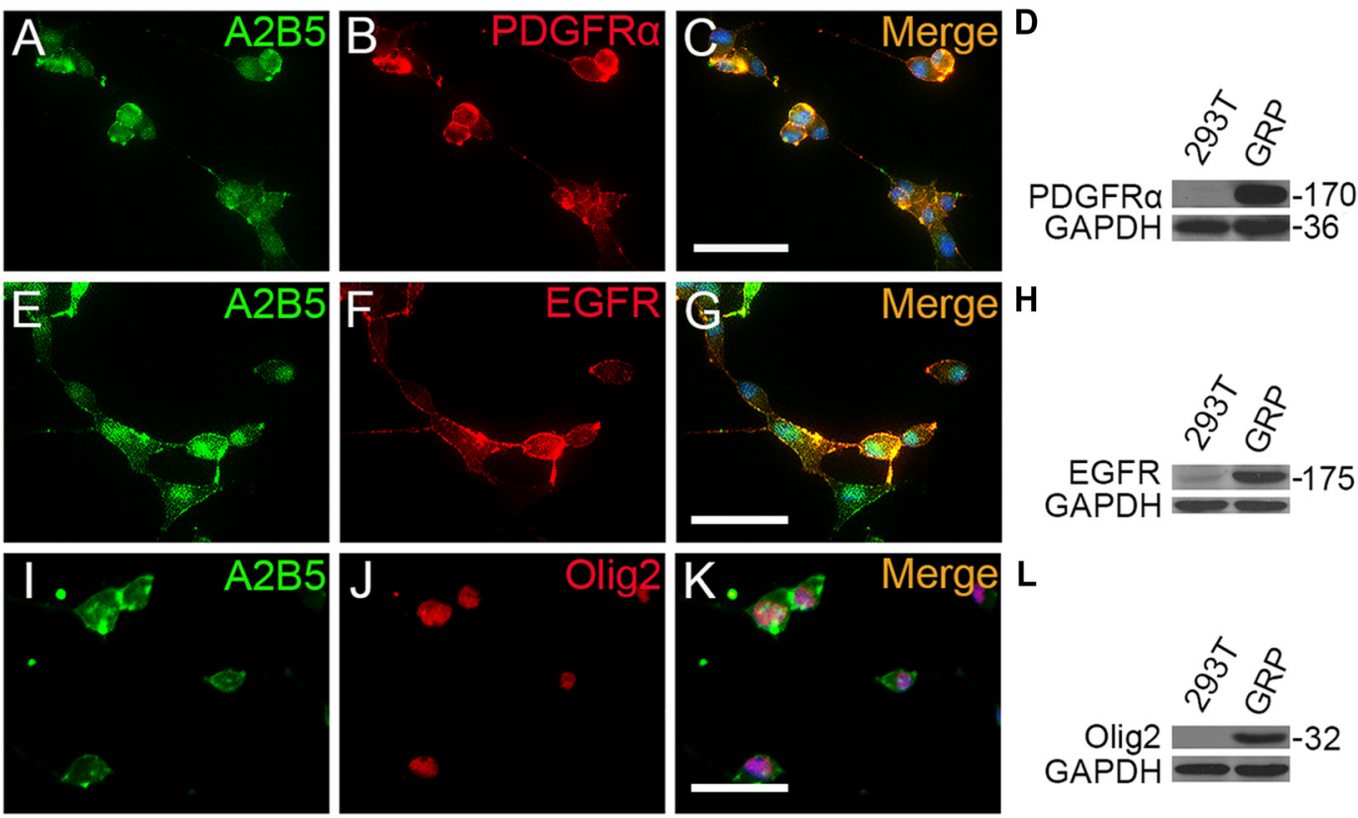

GAPDH $-175$
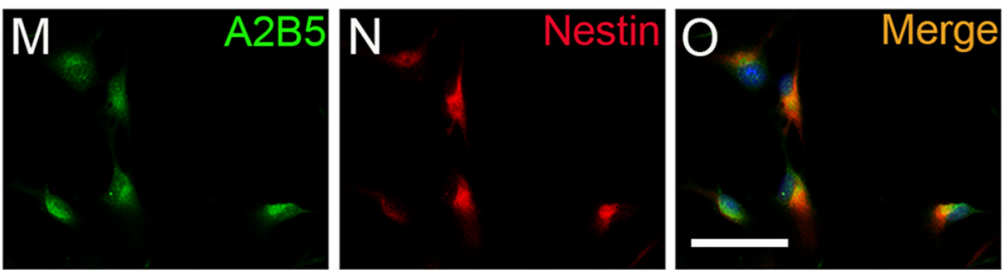

APDH

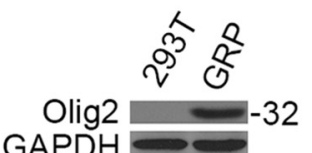

Q

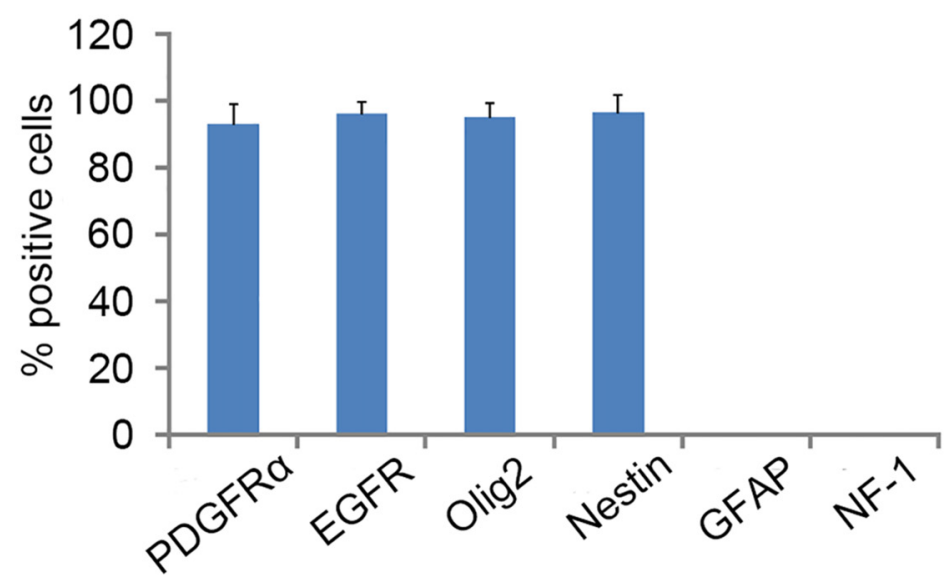

FIGURE 1 | The antigenic phenotypes of E13.5 spinal cord derived A2B5+ cells. (A-P) Immunostaining of A2B5+ cells with PDGFR $\alpha$, epidermal growth factor receptor (EGFR), Olig2 and Nestin antibodies, respectively, and the antigenic phenotypes were confirmed by Western blotting (D,H,L,P). (Q) Quantification of $\mathrm{PDGFR}^{+}, \mathrm{EGFR}^{+}, \mathrm{Olig}^{+}{ }^{+}, \mathrm{Nestin}^{+}, \mathrm{GFAP}^{+}$and NF-1 ${ }^{+}$cells in A2B5 ${ }^{+}$cell cultures, $n=3$. Scale bars: $50 \mu \mathrm{m}$

$\mathrm{O}^{+} / \mathrm{MBP}^{-}$cells $(1.3 \%+0.3 \%)$ were observed (Figure $\left.4 \mathrm{~B}\right)$. The specificity of EGF signaling in promoting oligodendrogenesis was confirmed with Erlotinib $\mathrm{HCl}$. When GRP cells were treated with EGF and Erlotinib $\mathrm{HCl}$ simultaneously, the antigenic phenotypes were similar to those of the controls (Figure 4B). Based on these results, we postulated that EGF plays a modest role in promoting the development of GRP cells into OPCs.
However, it is plausible that a considerable number of clones have differentiated into $\mathrm{O}^{-}$early-stage OPCs that could not be detected immunologically. To examine this possibility, we continued to culture these single GRP cell derived clones in glial basal medium for another 5 days in the absence of EGF. In the control group, only $1.5 \% \pm 0.2 \%$ of the cells were $\mathrm{MBP}^{+}$ (Figure 4D), similar to the percentage of $\mathrm{O}^{+}$clones prior to the 


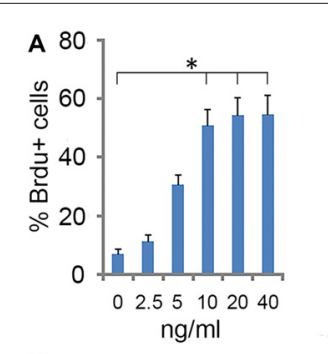

C
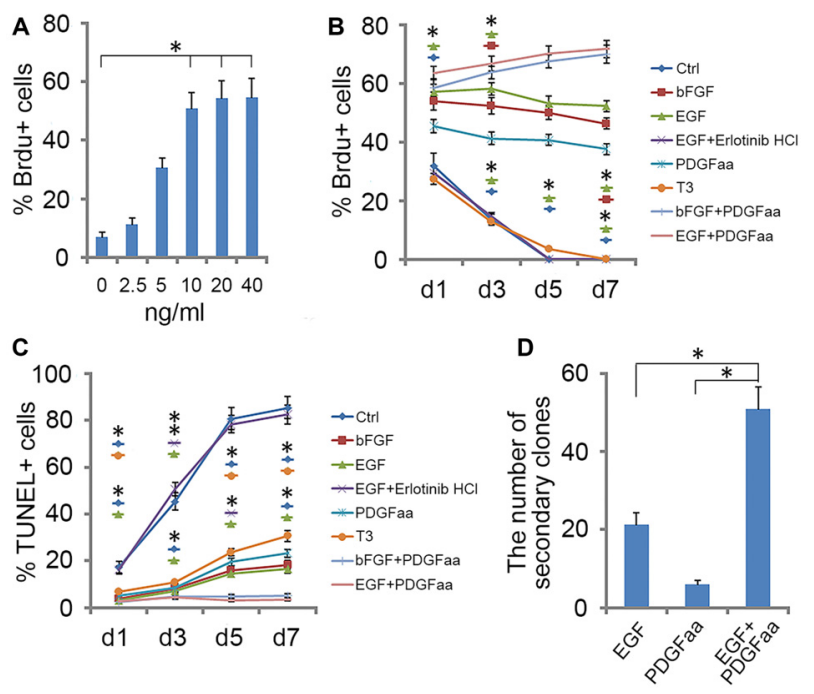

FIGURE 2 | Effect of EGF on A2B5 ${ }^{+}$cells. (A) Histogram of the number of $\mathrm{BrdU}^{+}$cells found in A2B5 ${ }^{+}$cell cultures after exposure to different doses of EGF. *Indicates differences between groups 10, 20 and $40 \mathrm{ng} / \mathrm{ml}$ EGF vs. the low-dose groups, $P<0.05$. No differences were found among the groups of 10, 20 and $40 \mathrm{ng} / \mathrm{ml} . n=3$. (B,C) Quantification of BrdU ${ }^{+}$and TUNEL ${ }^{+}$cells in $\mathrm{A}_{2} \mathrm{~B}^{+}{ }^{+}$cell cultures after EGF, EGF + Erlotinib-HCl, basic fibroblast growth factor (bFGF), PDGFaa, T3, bFGF + PDGFaa and EGF + PDGFaa treatments for various time lengths, Ctrl refers to the groups without any supplemented factor, $n=3$. (D) Histogram of the number of secondary clones in A2B5 ${ }^{+}$cell cultures at clonal density after exposure to EGF, PDGFaa and EGF + PDGFaa, respectively. $n=3$. Statistical analyses are presented as mean $\pm \mathrm{SD}$. ${ }^{*} P<0.05,{ }^{* *} P<0.01$.

5 -day culture (Figure 4B). In the EGF group, $32.4 \% \pm 2.7 \%$ of the clones generated $\mathrm{MBP}^{+}$cells (Figures 4C,D), suggesting that about $21.4 \%\left(32.4 \% \mathrm{MBP}^{+}-10.7 \% \mathrm{O}^{+} \mathrm{MBP}^{-}\right)$of the clones in EGF group generated $\mathrm{O}^{-}{ }^{-}$OPCs. Thus, EGF predisposed GRP cells to develop along OPCs.

However, EGF treatment alone was less efficient in fully transforming GRP cells into OPCs and maintaining their O4 negative antigen phenotype. We found that GRP cells exposed to a combination of EGF + PDGFaa + T3 exhibited a significant change from fibroblast morphology to bipolar or tripolar morphology, and remained $\mathrm{O} 4$ negative antigen phenotype. Moreover, the vast majority of clones $(89.1 \% \pm 3.7 \%)$ generated $\mathrm{O}^{+} / \mathrm{MBP}^{+} \mathrm{OLs}$ after growth factor withdrawal. This conjecture was further strengthened by their differentiation into $\mathrm{A}_{2} \mathrm{~B}^{+} / \mathrm{GFAP}^{+}$instead of $\mathrm{A} 2 \mathrm{~B}^{-} / \mathrm{GFAP}^{+}$astrocytes upon $\mathrm{FBS}$ treatment (Figure 4E), a bipotential differentiation characteristic of OPCs (Sommer and Schachner, 1981; Barnett et al., 1993).

\section{EGF Enhances the Self-Renewal of OPCs}

PDGFaa is an important mitogen for the self-renewal of OPCs (Noble et al., 1988; Raff et al., 1988; Hart et al., 1989; Neman and de Vellis, 2012), but it alone cannot maintain the $\mathrm{O} 4$ negative antigenic phenotype in OPCs. When GRP-derived early-stage $\mathrm{O}^{-}$OPCs from EGF + PDGFaa + T3 treatment were cultured in PDGFaa alone for 5 days, most cells became O4 positive $(88.7 \% \pm 5.9 \%$; Figure 5A) but MBP negative with few processes.
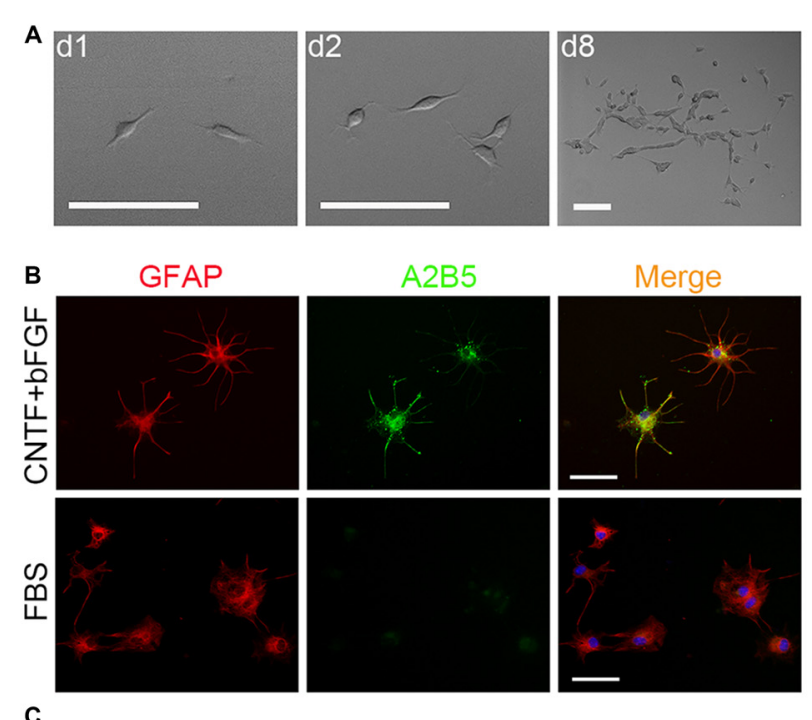

C
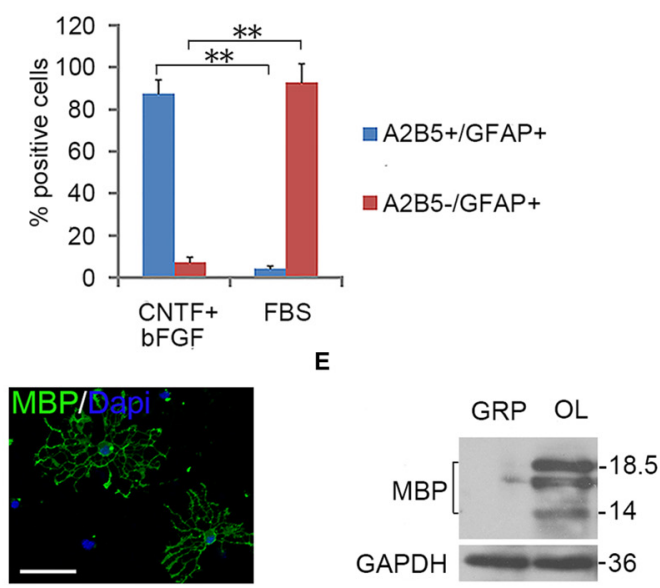

FIGURE 3 | Expanded clones of A2B5+ cell expressed typical differentiation phenotype of tripotential glial restricted precursor (GRP) cells. (A) An example of an expanded clone in presence of EGF + PDGFaa. (B) A2B5 ${ }^{+}$clones were digested and replated into separate wells of 24-well plates and induced to differentiate for 5 days in the presence of $\mathrm{bFGF}+\mathrm{CNTF}$ or FBS, A2B5+/GFAP ${ }^{+}$and A2B5-/GFAP + astrocytes were obtained, respectively. (C) Quantification of $\mathrm{A} 2 \mathrm{~B} 5^{+} / \mathrm{GFAP}+$ and $\mathrm{A}^{2} \mathrm{~B}^{-}{ }^{-} \mathrm{GFAP}^{+}$astrocytes in the differentiation cultures exposed to bFGF + CNTF and FBS, $n=3$. (D) A2B5 ${ }^{+}$cells were cultured in T3 for 5 days and $\mathrm{MBP}^{+}$Oligodendrocytes (OLs) can be detected. (E) The culture described in (D) was confirmed by western blotting with MBP antibody. Abbreviation: OL, oligodendrocyte. ${ }^{* *} P<0.01$. Scale bars: (A) $100 \mu \mathrm{m}$; (B,D) $50 \mu \mathrm{m}$.

These $\mathrm{O}^{+}$late-stage OPCs (Sommer and Schachner, 1981; Gard and Pfeiffer, 1990) continued to cycle (Figures 5A,C). When EGF was present, the majority of cells maintained bipolar or tripolar morphology, and the rate of $\mathrm{O}^{+}$cells decreased substantially $(7.4 \% \pm 2.6 \%)$ with a significant increase of $\mathrm{BrdU}^{+}$cells $(80 \% \pm 3.8 \%$; Figures $\mathbf{5 A} \mathbf{A}, \mathbf{B})$. Moreover, these expanded cultures of OPCs maintained by EGF + PDGFaa can differentiate into either mature $\mathrm{OLs}$ or $\mathrm{A} 2 \mathrm{~B}^{+} / \mathrm{GFAP}^{+}$astrocytes under specific culture conditions, indicating the differentiation potential was not compromised by proliferation, nor did they revert to GRP cells. Therefore, EGF have a synergistic effect 

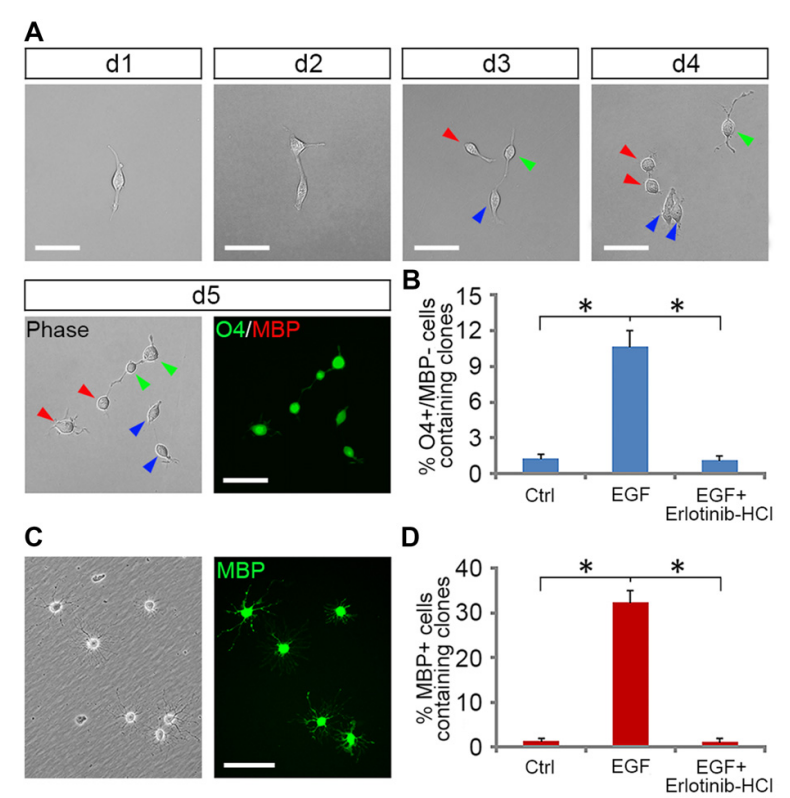

E
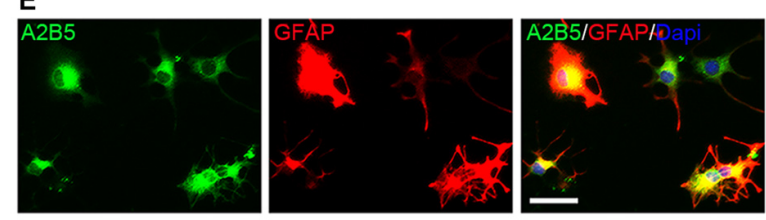

FIGURE 4 | EGF enhanced the formation of OPCs from GRP clones. (A) The single cell tracking of a GRP cell generating $\mathrm{O}^{+} / \mathrm{MBP}^{-}$daughter cells in EGF for 5 days. With the progression of cell division, a fibroblast-like GRP cell was gradually converted to typical OPCs of bipolar or tripolar morphology, well separated from each other, and expressed $\mathrm{O} 4$ antigen but not MBP. Daughter cells of the same progenitor were indicated by arrows of different color. (B) Quantification of the clones containing $\mathrm{O}^{+} / \mathrm{MBP}^{-}$cells in the GRP cell cultures exposed to various combinations of factors for 5 days, respectively, $n=3$. (C) OPC-like cells from (B) differentiated into $\mathrm{MBP}^{+}$cells after 5 days of culture in glial basal medium without supplemented factors. Left: phase image; right: anti-MBP immunostaining. (D) Quantification of the clones containing $\mathrm{MBP}^{+}$cells in $\mathrm{OL}$ differentiation cultures described in (C), $n=3$. (E) OPCs derived from EGF + PDGFaa + T3 treament differentiated into $\mathrm{A}^{2} \mathrm{~B}^{+} / \mathrm{GFAP}^{+}$astrocytes in presence of FBS. ${ }^{*} P<0.05$. Scale bars: (A,C) $75 \mu \mathrm{m} ;(\mathbf{E}) 50 \mu \mathrm{m}$.

with PDGFaa in the self-renewal of $\mathrm{O}^{-}$OPCs. And this cooperative effect of EGF and PDGFaa can be further amplified by other growth factor such as bFGF. When GRP-derived earlystage O4 $^{-}$OPCs were cultured in EGF + PDGFaa + bFGF in vitro, a faster cell division $(87.3 \% \pm 4.1 \%)$ and less $\mathrm{O}^{+}$ cells $(2.6 \% \pm 0.9 \%)$ were observed without compromising their differentiation characteristics, suggesting that the proliferation and self-renewal of OPCs are regulated by multiple signaling pathways.

\section{EGF Treatment Increased the Number of Differentiated OLs}

When GRP-derived $\mathrm{O}^{-}$OPCs were cultured in EGF alone, cells were initially active in proliferation, and many more BrdU-positive cells were found in EGF group than in control

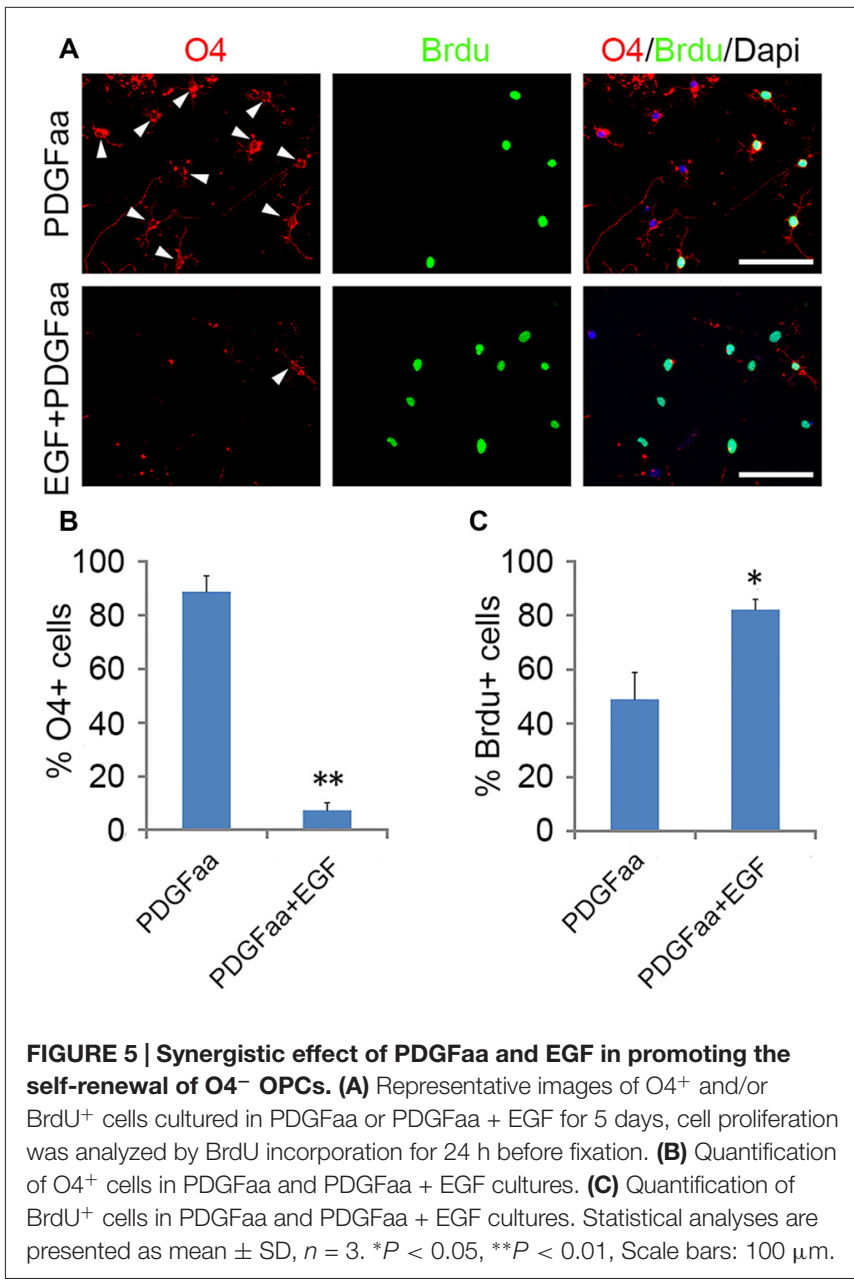

group. However, the percentage of $\mathrm{BrdU}^{+}$cells in EGF group decreased with time, and by day 4 , less than $3 \%$ of cells were proliferative (Figure 6A). Consistent with this, the EGFR expression was reduced more than half by day 4 (Figures 6D,E), which may partly contribute to the reduced effect of EGF on cell proliferation. It was found that bFGF cannot substitute for the loss of EGFR (data not shown), and the promotion of OPC proliferation by EGF or bFGF were based on the existence of PDGFaa. The reduced cell proliferation was accompanied by the increased proportion of $\mathrm{MBP}^{+}$cells in culture (Figure 6B). Although the rate of OPC differentiation under EGF treatment was slightly lower than that of control and T3 groups at the beginning, it increased rapidly on day 3 and exceeded the other two groups (Figure 6B), suggesting that EGF has a significant effect in promoting OL maturation when PDGFaa is not present. A synergistic effect was observed when T3 and EGF was combined, the differentiation was faster and the efficiency was higher than that of T3 alone or EGF alone (Figure 6B). TUNEL labeling experiments revealed that the rate of apoptosis with EGF treatment was significantly lower than that of the other two groups (Figure 6C). This raised the possibility that EGF treatment may partly increase the number of mature OLs indirectly by promoting their post-mitotic survival. 

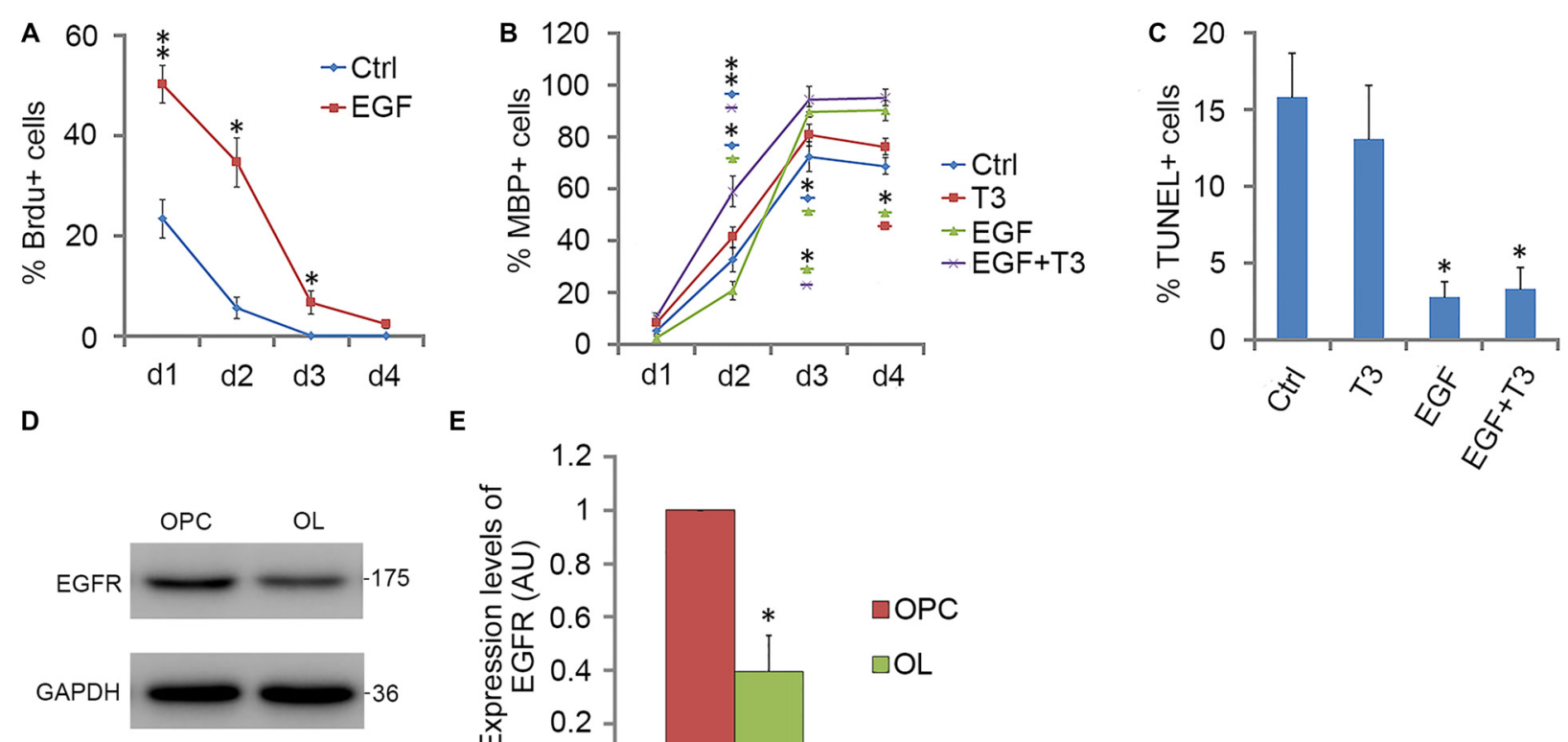

E

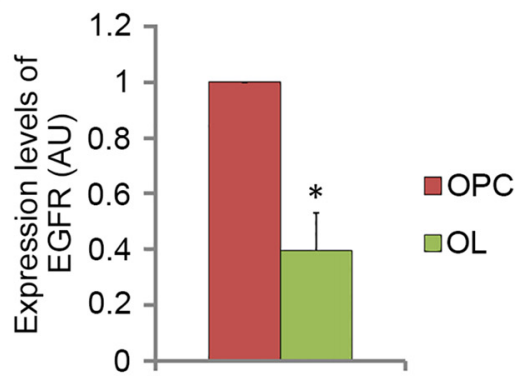

FIGURE 6 | EGF promotes OPC differentiation in cooperation with T3. (A) Quantification of $\mathrm{BrdU}^{+}$cells for OPCs cultures with EGF or not for various time lengths. (B) Quantification of $\mathrm{MBP}^{+}$cells in OPCs differentiation cultures treated with T3, EGF or T3 + EGF for various time lengths. (C) Quantification of TUNEL ${ }^{+}$ cells in OPCs differentiation cultures on day 4. (D,E) Western blotting and quantitative analysis of EGFR protein expression in $\mathrm{O}^{-}{ }^{-} \mathrm{OPCs}^{-}$and MBP+ $\mathrm{OLs}^{+}$, histograms express results in arbitrary units, taking GRP cells values as $100 \%$. Statistical analyses are presented as mean $\pm \mathrm{SD}, n=3 .{ }^{*} P<0.05,{ }^{* *} P<0.01$.

\section{DISCUSSION}

EGF is an extracellular signal molecule that binds to its specific receptor (EGFR) on the target cell membrane and then stimulates the phosphorylation of the receptor. The EGFR, also known as erbB1, is a glycoprotein that belongs to the related proteins family of c-erbB, there are three other members of this family: erbB2, erbB3 and erbB4 (Galvez-Contreras et al., 2013). The EGFR can generate homodimerization or heterodimerization with all ErbB family members, therefore, activated EGFR can stimulate a large number of downstream signaling molecules, and the complexity of its signal transduction determines the diversity of its biological effects. The diversity of EGF biological effects is reflected in the progression of OL lineage, as it plays distinct roles at different stages of OL development (Figure 7). The biological diversity of EGF signaling could be achieved by changing the balance between different signaling pathways (Tzahar et al., 1996).

\section{Role of EGF Signaling in GRP Development} GRP cells are probably the earliest progenitor cells for OL and astrocyte lineages (Rao et al., 1998; Gregori et al., 2002). GRP cells exhibited strong responsiveness to EGF for cell survival and proliferation in chemically defined medium. This result suggests that EGF signaling may be involved in the origin and expansion of GRP cells. Consistent with this idea, EGFR overexpression in postnatal white matter led to diffuse hyperplasia of progenitor cells that possess the same antigen phenotype as GRP cells in vitro (Ivkovic et al., 2008).

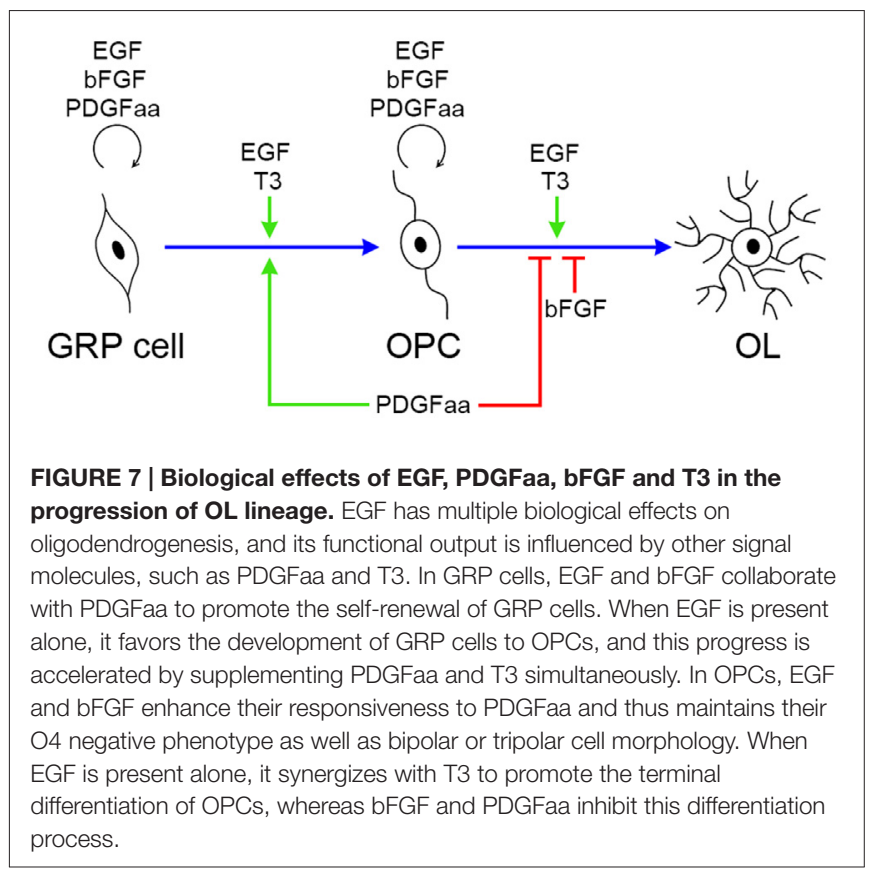

Moreover, following lysolecithin (LPC)-induced demyelination, a significantly higher number of GRP cells was observed in EGFR-overexpressing transgenic mice than that of the wild-type, leading to faster and more extensive remyelination, and more rapid functional recovery. Conversely, reduced EGFR signaling 
in vivo decreased the generation of OLs in postnatal brains (Aguirre et al., 2007).

EGF has a variety of biological effects on GRP cells, including cell survival and proliferation, and enhanced OL lineage progression. However, its eventual functional output is influenced by other signal molecules. For instance, PDGFaa and EGF work synergistically to maintain the self-renewal, the rate of cell proliferation decreased substantially after PDGFaa withdrawal, and GRP cells progressed into OPCs. The number of secondary clones in EGF group was much lower than that of PDGFaa + EGF group, probably due to the higher differentiation rate in the EGF group, and thus the reduced ability of GRP cells to form clones.

\section{Role of EGF Signaling in OPC Lineage Development}

OPCs retained strong responsiveness to EGF, and EGF enhanced the response of early-stage OPCs to PDGFaa in their self-renewal. In the absence of EGF, PDGFaa alone was difficult to prevent OPCs from expressing $\mathrm{O} 4$ antigen, and the cells then entered the secondary stage of OPC development with reduced proliferative capacity. The mechanisms underlying this enhanced response is not clear, one possibility is that it may function to maintain a high level of PDGFR $\alpha$ expression or signaling in OPCs (McKinnon et al., 1990). Thus, EGF may "set" a PDGF-driven clock in OPCs by establishing their sensitivity to PDGFaa (Galvez-Contreras et al., 2013). In this way, EGF may enlarge the OPC pool during CNS development.

The biological effect of EGF in promoting oligodendrogenesis was also reflected in the maturation of OPCs into OLs. EGF alone could not maintain the self-renewal of OPCs; instead it appeared to promote the differentiation of OPCs and the survival of mature OLs (Figure 6), leading to an increased number of $\mathrm{MBP}^{+}$mature OLs.

\section{Comparison of EGF and bFGF Effects in Oligodendroglial Lineage Development}

bFGF was commonly used in GRP cell culture, and it alone was sufficient to maintain the self-renewal of GRP cells in vitro (Gregori et al., 2002). Similarly, bFGF was also widely used in

\section{REFERENCES}

Aguirre, A., Dupree, J. L., Mangin, J. M., and Gallo, V. (2007). A functional role for EGFR signaling in myelination and remyelination. Nat. Neurosci. 10, 990-1002. doi: $10.1038 / \mathrm{nn} 1938$

Alizadeh, A., Dyck, S. M., and Karimi-Abdolrezaee, S. (2015). Myelin damage and repair in pathologic CNS: challenges and prospects. Front. Mol. Neurosci. 8:35. doi: 10.3389/fnmol.2015.00035

Bansal, R., and Pfeiffer, S. E. (1997). Regulation of oligodendrocyte differentiation by fibroblast growth factors. Adv. Exp. Med. Biol. 429, 69-77. doi: 10.1007/9781-4757-9551-6_5

Bansal, R., Stefansson, K., and Pfeiffer, S. E. (1992). Proligodendroblast antigen (POA), a developmental antigen expressed by A007/O4-positive oligodendrocyte progenitors prior to the appearance of sulfatide and galactocerebroside. J. Neurochem. 58, 2221-2229. doi: 10.1111/j.1471-4159. 1992.tb10967.x
OPC culture to suppress OPC differentiation and myelin gene expression (McKinnon et al., 1990; Bansal and Pfeiffer, 1997; Decker et al., 2000; Jiang et al., 2001). Thus, it seems that bFGF promotes progenitor maintenance by preventing the lineage progression of both GRP and OPC cells.

The present study has demonstrated that EGF is also effective in the maintenance of GRP and OPC culture. However, EGF appears to enhance the self-renewal of GRPs and OPCs by enhancing their responses to PDGFR $\alpha$ instead of directly inhibiting their differentiation. As a matter of fact, EGF treatment alone promotes the differentiation of both GRP cells and OPCs in culture. In the absence of PDGFaa and bFGF, EGF enhances GRP lineage progression to OPCs and OPC differentiation to mature OLs in synergy with T3 (Figure 7). Therefore, EGF and bFGF possess unique and distinct roles in oligodendroglial progenitor self-renewal and differentiation. Consistent with this notion, $b F G F^{-/-}$knockout leads to a higher proportion of mature OLs (Murtie et al., 2005), whereas EGFR mutation reduces the number of mature OLs (Aguirre et al., 2007).

\section{AUTHOR CONTRIBUTIONS}

JY designed, performed experiments, collected, analyzed the data and wrote the manuscript; $\mathrm{XC}, \mathrm{BX}, \mathrm{XZ}$ and $\mathrm{KZ}$ performed experiments; ZZ analyzed data; MQ designed, supervised the experiments, collected, analyzed and discussed data and wrote the manuscript. All authors listed, have made substantial, direct and intellectual contribution to the work, and approved it for publication.

\section{ACKNOWLEDGMENTS}

This work is supported by the National Key Basic Research Program of China (Ministry of Science and Technology of the People's Republic of China; 2013CB531303, 2012CB910402), National Natural Science Foundation of China (81200961, 31372150, 31572224, 31471955), the Zhejiang Provincial Natural Science Foundation of China (Y2111122, LQ16C090004), and the Medical and Health Research Program of Hangzhou in China (20170533B10).

Barnett, S. C., Franklin, R. J., and Blakemore, W. F. (1993). In vitro and in vivo analysis of a rat bipotential O-2A progenitor cell line containing the temperature-sensitive mutant gene of the SV40 large T antigen. Eur. J. Neurosci. 5, 1247-1260. doi: 10.1111/j.1460-9568.1993.tb00910.x

Blank, T., and Prinz, M. (2014). NF-кB signaling regulates myelination in the CNS. Front. Mol. Neurosci. 7:47. doi: 10.3389/fnmol.2014.00047

Cai, J., Zhu, Q., Zheng, K., Li, H., Qi, Y., Cao, Q., et al. (2010). Co-localization of Nkx6.2 and Nkx2.2 homeodomain proteins in differentiated myelinating oligodendrocytes. Glia 58, 458-468. doi: 10.1002/glia.20937

Cao, Q., Xu, X. M., Devries, W. H., Enzmann, G. U., Ping, P., Tsoulfas, P., et al. (2005). Functional recovery in traumatic spinal cord injury after transplantation of multineurotrophin-expressing glial-restricted precursor cells. J. Neurosci. 25, 6947-6957. doi: 10.1523/JNEUROSCI.1065-05.2005

Chen, Y., Balasubramaniyan, V., Peng, J., Hurlock, E. C., Tallquist, M., Li, J., et al. (2007). Isolation and culture of rat and mouse oligodendrocyte precursor cells. Nat. Protoc. 2, 1044-1051. doi: 10.1038/nprot.2007.149 
Chen, Y., Mei, R., Teng, P., Yang, A., Hu, X., Zhang, Z., et al. (2015). TAPP1 inhibits the differentiation of oligodendrocyte precursor cells via suppressing the Mek/Erk pathway. Neurosci. Bull. 31, 517-526. doi: 10.1007/s12264-015-1537-5

Cheng, X., Xie, B., Qi, J., Zhao, X., Zhang, Z., Qiu, M., et al. (2017). Rat astrocytes are more supportive for mouse OPC self-renewal than mouse astrocytes in culture. Dev. Neurobiol. doi: 10.1002/dneu.22476 [Epub ahead of print].

Chong, V. Z., Webster, M. J., Rothmond, D. A., and Weickert, C. S. (2008). Specific developmental reductions in subventricular zone ErbB1 and ErbB4 mRNA in the human brain. Int. J. Dev. Neurosci. 26, 791-803. doi: 10.1016/j.ijdevneu. 2008.06.004

Crang, A. J., Gilson, J. M., Li, W.-W., and Blakemore, W. F. (2004). The remyelinating potential and in vitro differentiation of MOG-expressing oligodendrocyte precursors isolated from the adult rat CNS. Eur. J. Neurosci. 20, 1445-1460. doi: 10.1111/j.1460-9568.2004.03606.x

Dadsetan, M., Knight, A. M., Lu, L., Windebank, A. J., and Yaszemski, M. J. (2009). Stimulation of neurite outgrowth using positively charged hydrogels. Biomaterials 30, 3874-3881. doi: 10.1016/j.biomaterials.2009.04.018

Decker, L., Avellana-Adalid, V., Nait-Oumesmar, B., Durbec, P., and BaronVan, Evercooren, A. (2000). Oligodendrocyte precursor migration and differentiation: combined effects of PSA residues, growth factors, and substrates. Mol. Cell. Neurosci. 16, 422-439. doi: 10.1006/mcne. 2000.0885

Dincman, T. A., Beare, J. E., Ohri, S. S., and Whittemore, S. R. (2012). Isolation of cortical mouse oligodendrocyte precursor cells. J. Neurosci. Methods. 209, 219-226. doi: 10.1016/j.jneumeth.2012.06.017

Fernandez, M., Pirondi, S., Manservigi, M., Giardino, L., and Calzà, L. (2004). Thyroid hormone participates in the regulation of neural stem cells and oligodendrocyte precursor cells in the central nervous system of adult rat. Eur. J. Neurosci. 20, 2059-2070. doi: 10.1111/j.1460-9568.2004.03664.x

Galvez-Contreras, A. Y., Quiñones-Hinojosa, A., and Gonzalez-Perez, O. (2013). The role of EGFR and ErbB family related proteins in the oligodendrocyte specification in germinal niches of the adult mammalian brain. Front. Cell. Neurosci. 7:258. doi: 10.3389/fncel.2013.00258

Gard, A. L., and Pfeiffer, S. E. (1990). Two proliferative stages of the oligodendrocyte lineage (A2B5+O4- and O4+GalC-) under different mitogenic control. Neuron 5, 615-625. doi: 10.1016/0896-6273(90)90216-3

Gonzalez-Perez, O., Romero-Rodriguez, R., Soriano-Navarro, M., GarciaVerdugo, J. M., and Alvarez-Buylla, A. (2009). Epidermal growth factor induces the progeny of subventricular zone type B cells to migrate and differentiate into oligodendrocytes. Stem Cells 27, 2032-2043. doi: 10.1002/stem.119

Gregori, N., Pröschel, C., Noble, M., and Mayer-Pröschel, M. (2002). The tripotential glial-restricted precursor (GRP) cell and glial development in the spinal cord: generation of bipotential oligodendrocyte-type- 2 astrocyte progenitor cells and dorsal-ventral differences in GRP cell function. J. Neurosci. 22, 248-256.

Haas, C., Neuhuber, B., Yamagami, T., Rao, M., and Fischer, I. (2012). Phenotypic analysis of astrocytes derived from glial restricted precursors and their impact on axon regeneration. Exp. Neurol. 233, 717-732. doi: 10.1016/j.expneurol. 2011.11.002

Hart, I. K., Richardson, W. D., Heldin, C. H., Westermark, B., and Raff, M. C. (1989). PDGF receptors on cells of the oligodendrocyte-type-2 astrocyte (O-2A) cell lineage. Development 105, 595-603.

Herrera, J., Yang, H., Zhang, S. C., Proschel, C., Tresco, P., Duncan, I. D., et al. (2001). Embryonic-derived glial-restricted precursor cells (GRP cells) can differentiate into astrocytes and oligodendrocytes in vivo. Exp. Neurol. 171, 11-21. doi: 10.1006/exnr.2001.7729

Hill, C. E., Proschel, C., Noble, M., Mayer-Proschel, M., Gensel, J. C., Beattie, M. S., et al. (2004). Acute transplantation of glial-restricted precursor cells into spinal cord contusion injuries: survival, differentiation and effects on lesion environment and axonal regeneration. Exp. Neurol. 190, 289-310. doi: 10.1016/j.expneurol.2004.05.043

Hu, Q., Zhang, L., Wen, J., Wang, S., Li, M., Feng, R., et al. (2010). The EGF receptor-sox2-EGF receptor feedback loop positively regulates the self-renewal of neural precursor cells. Stem Cells 28, 279-286. doi: 10.1002/ stem. 246

Ivkovic, S., Canoll, P., and Goldman, J. E. (2008). Constitutive EGFR signaling in oligodendrocyte progenitors leads to diffuse hyperplasia in postnatal white matter. J. Neurosci. 28, 914-922. doi: 10.1523/JNEUROSCI.4327-07.2008
Jiang, F., Frederick, T. J., and Wood, T. L. (2001). IGF-I synergizes with FGF-2 to stimulate oligodendrocyte progenitor entry into the cell cycle. Dev. Biol. 232, 414-423. doi: 10.1006/dbio.2001.0208

Liu, Y., Wu, Y., Lee, J. C., Xue, H., Pevny, L. H., Kaprielian, Z., et al. (2002). Oligodendrocyte and astrocyte development in rodents: an in situ and immunohistological analysis during embryonic development. Glia 40, 25-43. doi: 10.1002/glia.10111

McKinnon, R. D., Matsui, T., Dubois-Dalcq, M., and Aaronson, S. A. (1990). FGF modulates the PDGF-driven pathway of oligodendrocyte development. Neuron 5, 603-614. doi: 10.1016/0896-6273(90)90215-2

Morath, D. J., and Mayer-Pröschel, M. (2001). Iron modulates the differentiation of a distinct population of glial precursor cells into oligodendrocytes. Dev. Biol. 237, 232-243. doi: 10.1006/dbio.2001.0352

Murtie, J. C., Zhou, Y. X., Le, T. Q., and Armstrong, R. C. (2005). In vivo analysis of oligodendrocyte lineage development in postnatal FGF2 null mice. Glia 49, 542-554. doi: 10.1002/glia.20142

Neman, J., and de Vellis, J. (2012). A method for deriving homogenous population of oligodendrocytes from mouse embryonic stem cells. Dev. Neurobiol. 72, 777-788. doi: 10.1002/dneu.22008

Noble, M., Murray, K., Stroobant, P., Waterfield, M. D., and Riddle, P. (1988). Platelet-derived growth factor promotes division and motility and inhibits premature differentiation of the oligodendrocyte/type- 2 astrocyte progenitor cell. Nature 333, 560-562. doi: 10.1038/333560a0

Phillips, A. W., Falahati, S., DeSilva, R., Shats, I., Marx, J., Arauz, E., et al. (2012). Derivation of glial restricted precursors from E13 mice. J. Vis. Exp. 64:3462. doi: $10.3791 / 3462$

Qiu, M. (2013). Myelin in development and disease. Neurosci. Bull. 29, 127-128. doi: 10.1007/s12264-013-1325-Z

Raff, M. C., Lillien, L. E., Richardson, W. D., Burne, J. F., and Noble, M. D. (1988). Platelet-derived growth factor from astrocytes drives the clock that times oligodendrocyte development in culture. Nature 333, 562-565. doi: $10.1038 / 333562 \mathrm{a} 0$

Rao, M. S., Noble, M., and Mayer-Pröschel, M. (1998). A tripotential glial precursor cell is present in the developing spinal cord. Proc. Natl. Acad. Sci. U S A 95, 3996-4001. doi: 10.1073/pnas.95.7.3996

Rao, S. N., and Pearse, D. D. (2016). Regulating axonal responses to injury: the intersection between signaling pathways involved in axon myelination and the inhibition of axon regeneration. Front. Mol. Neurosci. 9:33. doi: 10.3389/fnmol. 2016.00033

Rodríguez-Peña, A. (1999). Oligodendrocyte development and thyroid hormone. J. Neurobiol. 40, 497-512. doi: 10.1002/(SICI)10974695(19990915)40:4<497::AID-NEU7>3.0.CO;2-\#

Sántha, P., Veszelka, S., Hoyk, Z., Mészáros, M., Walter, F. R., Tóth, A. E., et al. (2016). Restraint stress-induced morphological changes at the bloodbrain barrier in adult rats. Front. Mol. Neurosci. 8:88. doi: 10.3389/fnmol.2015. 00088

Scafidi, J., Hammond, T. R., Scafidi, S., Ritter, J., Jablonska, B., Roncal, M., et al. (2014). Intranasal epidermal growth factor treatment rescues neonatal brain injury. Nature 506, 230-234. doi: 10.1038/nature12880

Shaw, G., Osborn, M., and Weber, K. (1981). An immunofluorescence microscopical study of the neurofilament triplet proteins, vimentin and glial fibrillary acidic protein within the adult rat brain. Eur. J. Cell Biol. 26, 68-82.

Sommer, I., and Schachner, M. (1981). Monoclonal antibodies (O1 to O4) to oligodendrocyte cell surfaces: an immunocytological study in the central nervous system. Dev. Biol. 83, 311-327. doi: 10.1016/0012-1606(81) 90477-2

Sun, J. J., Liu, Y., and Ye, Z. R. (2008). Effects of P2Y1 receptor on glial fibrillary acidic protein and glial cell line-derived neurotrophic factor production of astrocytes under ischemic condition and the related signaling pathways. Neurosci. Bull. 24, 231-243. doi: 10.1007/s12264-008-0430-x

Tang, J., Xu, H. W., Fan, X. T., Li, Z. F., Li, D. B., Yang, L., et al. (2007). Targeted migration and differentiation of engrafted neural precursor cells in amyloid $\beta$ treated hippocampus in rats. Neurosci. Bull. 23, 263-270. doi: 10.1007/s12264007-0039-5

Tzahar, E., Waterman, H., Chen, X., Levkowitz, G., Karunagaran, D., Lavi, S., et al. (1996). A hierarchical network of interreceptor interactions determines signal transduction by Neu differentiation factor/neuregulin and epidermal growth factor. Mol. Cell Biol. 16, 5276-5287. doi: 10.1128/mcb.16.10.5276 
Wu, Y. Y., Mujtaba, T., Han, S. S., Fischer, I., and Rao, M. S. (2002). Isolation of a glial-restricted tripotential cell line from embryonic spinal cord cultures. Glia 38, 65-79. doi: 10.1002/glia.10049

Yang, J., Cheng, X., Shen, J., Xie, B., Zhao, X., Zhang, Z., et al. (2016). A novel approach for amplification and purification of mouse oligodendrocyte progenitor cells. Front. Cell. Neurosci. 10:203. doi: 10.3389/fncel.2016.00203

Yang, J., Liu, X., Yu, J., Sheng, L., Shi, Y., Li, Z., et al. (2009). A non-viral vector for potential DMD gene therapy study by targeting a minidystrophin-GFP fusion gene into the hrDNA locus. Acta Biochim. Biophys. Sin. (Shanghai) 41, 1053-1060. doi: 10.1093/abbs/gmp080

Yoo, S., and Wrathall, JR. (2007). Mixed primary culture and clonal analysis provide evidence that NG2 proteoglycan-expressing cells after spinal cord injury are glial progenitors. Dev. Neurobiol. 67, 860-874. doi: 10.1002/dneu. 20369

Zhang, Y., Guo, T. B., and Lu, H. (2013). Promoting remyelination for the treatment of multiple sclerosis: opportunities and challenges. Neurosci. Bull. 29, 144-154. doi: 10.1007/s12264-013-1317-z
Zhao, J. W., Raha-Chowdhury, R., Fawcett, J. W., and Watts, C. (2009). Astrocytes and oligodendrocytes can be generated from $\mathrm{NG}^{+}$progenitors after acute brain injury: intracellular localization of oligodendrocyte transcription factor 2 is associated with their fate choice. Eur. J. Neurosci. 29, 1853-1869. doi: $10.1111 /$ j.1460-9568.2009.06736.x

Conflict of Interest Statement: The authors declare that the research was conducted in the absence of any commercial or financial relationships that could be construed as a potential conflict of interest.

Copyright (c) 2017 Yang, Cheng, Qi, Xie, Zhao, Zheng, Zhang and Qiu. This is an open-access article distributed under the terms of the Creative Commons Attribution License (CC BY). The use, distribution or reproduction in other forums is permitted, provided the original author(s) or licensor are credited and that the original publication in this journal is cited, in accordance with accepted academic practice. No use, distribution or reproduction is permitted which does not comply with these terms. 